\title{
Dí Strueretllámung
}

\section{nad) dem nRuRn \\ bayeríd)en Gewerb/teuergelet}

Nit ausgefüllten $\mathfrak{N u f t e r f o r m u l a r e n ~ u n b ~ E ̂ t l a ̈ u t e r u n g e n . ~}$

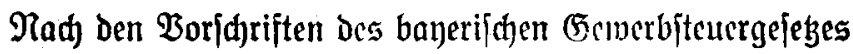
vom 9. Inll 1926 bearbeitet

von

Dr. Rudolf Wallermann

Redtizanwalt und Snıbikus in Münd)en.

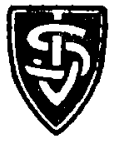

1927

Münden - Berlín / Eeipzíg

J. 秥weifer berlag (Frthur Gellier). 



\section{Die Steureretlánung}

nad) dem $n \in \mathbb{R} \mathbf{R}$

\section{bayeri/d)en Gewerb/teuergelets}

Mit ausgefüllten Mufterformularen und $\mathfrak{E}$ rläuterungen.

Tad Den Borjdriften Des bayeri|den (Gemerbjteuergejeges vom 9. Suli 1926 bearbeitet

voll

\section{Dr. Kudolf $\mathfrak{w a}$ alfermann}

Red)tsantoalt und Sqndikus in Mündelt.

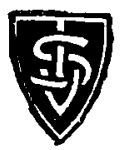

1927

Mündyen - Berlín / Eeipzíg

J. Sdweitger berlag (Arthur Sellíer). 